\title{
Nucleosomal histone kinase-1 phosphorylates H2A Thr 119 during mitosis in the early Drosophila embryo
}

\author{
Hitoshi Aihara, ${ }^{1}$ Takeya Nakagawa, ${ }^{1}$ Kiyoshi Yasui, ${ }^{1}$ Tsutomu Ohta, ${ }^{2}$ Susumu Hirose, ${ }^{3}$ \\ Naoshi Dhomae, ${ }^{4}$ Koji Takio, ${ }^{5}$ Mayumi Kaneko, ${ }^{6}$ Yukio Takeshima, ${ }^{6}$ Masami Muramatsu, ${ }^{7}$ and \\ Takashi Ito ${ }^{1,8}$ \\ ${ }^{1}$ Department of Biochemistry, Nagasaki University School of Medicine, Nagasaki, Nagasaki 852-8523, Japan; ${ }^{2}$ Medical \\ Genomic Center, National Cancer Center Research Institute, Tokyo 104-0045, Japan; ${ }^{3}$ Department of Developmental \\ Genetics, National Institute of Genetics, Shizuoka-ken 411-8540, Japan; ${ }^{4}$ The Institute of Physical and Chemical Research, \\ Wako-shi, Saitama 351-0198, Japan; ${ }^{5}$ RIKEN Harima Institute, Mikazuki-cho, Hyogo 679-5148, Japan; ${ }^{6}$ Second Department \\ of Pathology, Hiroshima University School of Medicine, Minami-ku, Hiroshima 734-8551, Japan; ${ }^{7}$ Saitama Medical School \\ Research Center for Genomic Medicine, Hidaka, Saitama 350-1241, Japan
}

\begin{abstract}
Posttranslational histone modifications are important for the regulation of many biological phenomena. Here, we show the purification and characterization of nucleosomal histone kinase-1 (NHK-1). NHK-1 has a high affinity for chromatin and phosphorylates a novel site, Thr 119, at the C terminus of H2A. Notably, NHK-1 specifically phosphorylates nucleosomal H2A, but not free H2A in solution. In Drosophila embryos, phosphorylated H2A Thr 119 is found in chromatin, but not in the soluble core histone pool. Immunostaining of NHK-1 revealed that it goes to chromatin during mitosis and is excluded from chromatin during $S$ phase. Consistent with the shuttling of NHK-1 between chromatin and cytoplasm, H2A Thr 119 is phosphorylated during mitosis but not in $\mathrm{S}$ phase. These studies reveal that NHK-1-catalyzed phosphorylation of a conserved serine/threonine residue in $\mathrm{H} 2 \mathrm{~A}$ is a new component of the histone code that might be related to cell cycle progression.
\end{abstract}

[Keywords: Nucleosome; phosphorylation; histone code; transcription; NHK-1]

Supplemental material is available at http://genesdev.org.

Received January 6, 2004; revised version accepted March 15, 2004

The eukaryotic genome is packaged into chromatin, which not only constrains the genome within the boundaries of the cell nucleus but also permits dynamic and broad-ranging changes related to many important biological phenomena (for review, see Kornberg and Lorch 1999; Vignali et al. 2000; Narlikar et al. 2002; Orphanides and Reinberg 2002; Ito 2003; Martens and Winston 2003). Diverse posttranslational modifications of the core histones occur, often on the tail domains (for review, see Zhang and Reinberg 2001; Carrozza et al. 2003; Fischle et al. 2003; Kurdistani and Grunstein 2003). Among these posttranslational modifications, histone acetylation has been one of the most studied, in part because of the discovery of histone acetyltransferases and histone deacetylases, which are responsible for bringing about the steady-state balance of these modifications (for review, see Strahl and Allis 2000).

In recent years, however, research has shown that phosphorylation of histone proteins is important in the

${ }^{8}$ Corresponding author.

E-MAIL tito@net.nagasaki-u.ac.jp; FAX 81-95-849-7040.

Article published online ahead of print. Article and publication date are at http://www.genesdev.org/cgi/doi/10.1101/gad.1184604. regulation of many phenomena in the nucleus, and enzymes that regulate these modifications have also been identified. For example, phosphorylation of Ser 10 in histone $\mathrm{H} 3$ is regulated by several kinases, such as IKK- $\alpha$ (Anest et al. 2003; Yamamoto et al. 2003), Rsk-2 (Sassone-Corsi et al. 1999), and Snf1 (Lo et al. 2001). Phosphorylation of Ser 10 in core histone H3 correlates with chromosome condensation during mitosis (Hsu et al. 2000). It was suspected that H3 Ser 10 phosphorylation may be a signal that drives dynamic chromosome condensation or may itself alter chromatin structure (Cheung et al. 2000; Lo et al. 2000). In independent pathways, H3 Ser 10 phosphorylation is synergistically coupled to acetylation of Lys 14, resulting in stimulation of gene expression (Cheung et al. 2000; Lo et al. 2000). Ser 139 in H2A.X, a histone H2A variant, is phosphorylated by ATR kinase in response to DNA damage and may cause an alteration of chromatin structure that facilitates DNA repair (Downs et al. 2000; Rogakou et al. 2000; Ward and Chen 2001). Recently, phosphorylation of Ser 14 in histone H2B regulated by Mstl kinase was found to be induced in apoptotic cells (Cheung et al. 2003). In addition to these sites, more phosphorylation sites might be important (Goll and Bestor 2002). The 
biological functions of these histone phosphorylations and the enzymes that regulate these phosphorylation sites remain to be elucidated. The importance of phosphorylation of histone proteins led us to investigate histone kinases that regulate important biological phenomena. In this study, we found a novel nucleosomal kinase activity that catalyzes phosphorylation of a conserved serine/threonine residue in $\mathrm{H} 2 \mathrm{~A}$ only in the context of the nucleosome. In addition, we demonstrated that this phosphorylation site is a new component of the histone code related to cell cycle progression.

\section{Results}

Purification and identification of nucleosomal histone kinase-1, NHK-1

We fractionated nuclear extract derived from Drosophila embryos and found a novel kinase activity. A kinase assay with free core histones as substrates using fractions from Q Sepharose chromatography is shown in Figure 1A. The results suggest that several kinases phosphorylate free core histones. However, when using chromatin as a substrate, it was shown that there was only one kinase acting in the flow-through fraction as indicated by phosphorylation of histone H2A-H2B (Fig. 1A, right panel, indicated by asterisk). The kinase activity that phosphorylated H2A-H2B in nucleosome form did not phosphorylate solubilized free core histones (Fig. 1A, left panel, indicated by asterisk). Further purification was undertaken to identify this unique kinase and to elucidate the role of this phosphorylation (the steps in purification are depicted in Fig. 1B).

We examined this unique activity through purification monitoring by kinase assay using chromatin. Fractions from the final HiTrap Blue chromatography step were analyzed by SDS-polyacrylamide gel electrophoresis (SDS-PAGE) and subsequent staining with Coomassie Brilliant Blue R-250 (Fig. 1C). Three polypeptides with molecular masses of 70,52 , and $42 \mathrm{kD}$ (indicated by asterisks in Fig. 1C) were copurified with nucleosomal histone kinase (NHK) activity. These polypeptide bands were in-gel digested with lysylendopeptidase and subjected to HPLC fractionation. Partial amino acid sequences were determined by Edman peptide analysis. Two sequences derived from these three polypeptides (EGTVFTDLAK and AANILLGLEK) were found to be identical to the segments of the Drosophila CG6386 gene product with an apparent serine/threonine kinase domain (see FlyBase report; http://flybase.bio.indiana. edu).

To exclude the possibility that another kinase is included in this fraction, we performed LC-tandem-mass analysis using the SDS gel fragments sliced from top to bottom. No other predicted kinases in Drosophila except CG6386 were present in these samples. Thus, we named this protein kinase nucleosomal histone kinase1, NHK-1.

$N H K-1$ is a novel gene that encodes a protein of 599 amino acids. By Western blot analysis with anti-NHK-1 antibodies using Drosophila extract, a $70-\mathrm{kD}$ band is detected throughout Drosophila development, with the highest level in early embryos (data not shown). Because its calculated molecular mass is $65.993 \mathrm{kD}$, two of the three bands observed during purification might be proteolyzed products.

This kinase is substrate-specific. Highly purified NHK-1 did not phosphorylate purified H2A-H2B dimer in the presence or absence of $\mathrm{H} 3-\mathrm{H} 4$ tetramer. However, NHK-1 phosphorylated nucleosomal H2A-H2B dramatically. NHK-1 phosphorylated $\mathrm{H} 2 \mathrm{~A}-\mathrm{H} 2 \mathrm{~B}$ in the presence of DNA, but to a much lesser extent than in the nucleosome (Fig. 1D).

\section{Purified recombinant NHK-1 has nucleosomal histone kinase activity}

To conclude that NHK-1 is really a nucleosomal histone kinase, we undertook synthesis and purification of the recombinant proteins. To this end, we constructed bacterial expression vectors for full-length NHK-1 with N-terminal tandem Flag epitope tag (termed Flag-NHK-1). Flag-NHK-1 was expressed in Escherichia coli and purified by affinity purification via the Flag epitope tag (Fig. 2A), and its kinase activity was tested. Recombinant Flag-NHK-1 also phosphorylates only nucleosomal H2A-H2B, as does native NHK-1 (Fig. 2B). These results confirm the conclusion that the purified kinase is a nucleosome-specific histone kinase.

We generated rabbit polyclonal anti-NHK-1. Wholeembryo extracts before gastrulation (0 3 h embryo) and after gastrulation (3 6 h embryo) were run together with Flag-NHK-1 and subjected to subsequent immunoblotting with anti-NHK-1. The molecular size of the native NHK-1 is $70 \mathrm{kD}$, based on the immunoblotting of wholecell extract. As a control, dNLP was used for estimation of the total protein (Ito et al. 1996a). Flag-NHK-1 ran more slowly than native NHK-1 partially because of the tandem Flag tag (Fig. 2C).

Comparison of the predicted amino acid sequence of this unique NHK-1 with known proteins revealed similarities to other known kinases, such as human Vaccinia related kinase 1 (VRK1; Nezu et al. 1997), mouse VRK1 (GenBank accession no. AAH16676), Xenopus VRK (GenBank accession no. AAH41230), and Caenorhabditis elegans VRK (2G213), with conservation of $44 \%$, $43 \%, 41 \%$, and $37 \%$ of this kinase domain, respectively (Fig. 2D). In addition to a conserved kinase domain, there is common structure, namely, an acidic amino acid region between the basic amino acid regions. This basic-acidic-basic amino acid motif (BAB motif) is conserved among species, as illustrated in Figure 2E. This motif may prove interesting for future crystallographic studies.

Concerning the functions of VRK1, it has been reported that human VRK1 phosphorylates Thr 18 of p53, the binding site of $\mathrm{mdm} 2$. However, the biological role of human VRK1, like that of NHK-1, is not known (Lopez- 


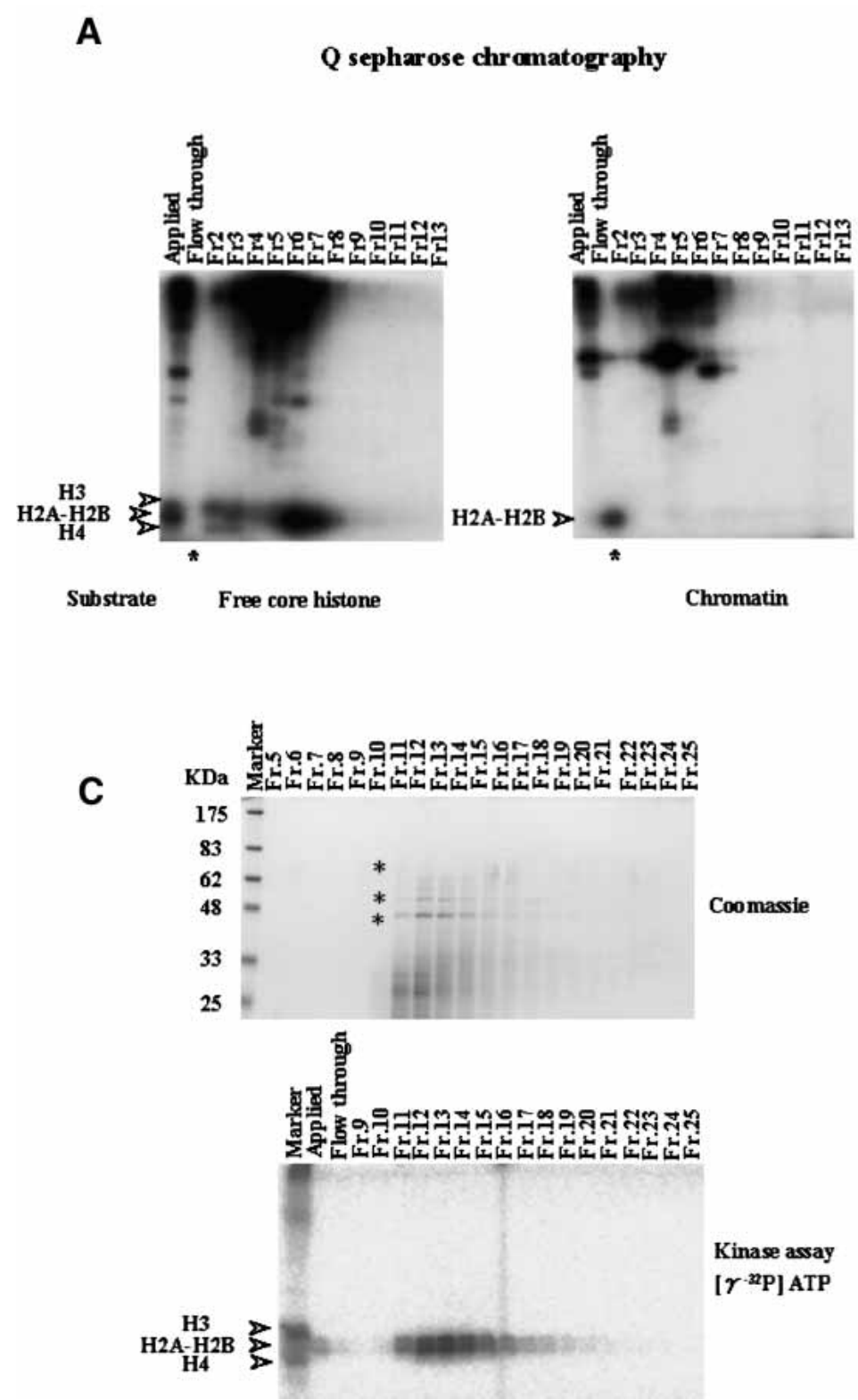

B

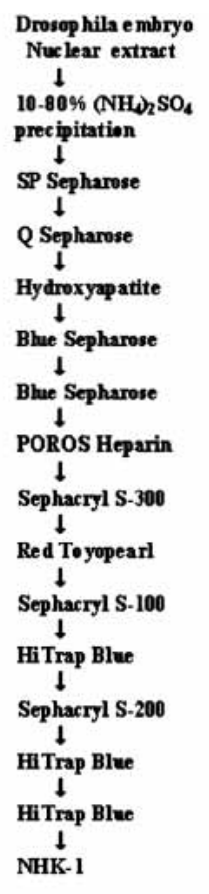

D

+Native NHK-1
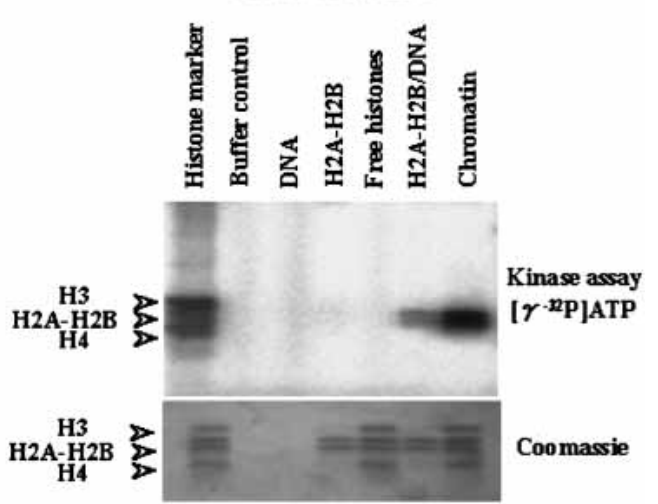

Figure 1. Identification and purification of nucleosomal histone kinase-1 (NHK-1). (A) Kinase assay from Q Sepharose chromatography (see $B)$. Fractions were incubated with free core histones or chromatin as substrates in the presence of $\left[\gamma^{-32} \mathrm{P}\right] \mathrm{ATP}$. These reaction mixtures were run on $15 \%$ SDS-PAGE and analyzed by autoradiography. Asterisks indicate initial identification of nucleosomal histone kinase activity. (B) Scheme for purification of NHK-1. (C, bottom panel) The kinase assay using both chromatin as substrate and fractions from the final HiTrap blue column chromatography (see $B$ ). (Top panel) These same fractions were precipitated with TCA, run on 10\% SDS-PAGE, and stained with Coomassie Brilliant Blue. The p70, p52, and p42 bands indicated by asterisks were subjected to peptide sequencing. $(D)$ Kinase assay used highly purified native NHK-1 in the presence of DNA, H2A-H2B dimer, all four core histones, H2A-H2B/DNA, or chromatin as substrates. All four core histones were phosphorylated by nonspecific kinase and used as histone markers.

Borges and Lazo 2000). C. elegans VRK was characterized in a systematic analysis of the $C$. elegans genome using RNAi. Inactivation of C. elegans VRK by RNAi revealed embryonic lethality, with large cytoplasmic granules and failure to form a pronucleus (Piano et al. 2002; Kamath et al. 2003). These reports suggest that VRK has important functions for viability.
NHK-1 can phosphorylate Thr 119 in nucleosomal H2A but not in free H2A

We next examined which amino acid residues are phosphorylated by NHK-1. First, nucleosomal histones were phosphorylated by native NHK-1 in the presence of $\left[\gamma^{32} \mathrm{P}\right] \mathrm{ATP}$ and run on SDS gels. Labeled bands were di- 
A

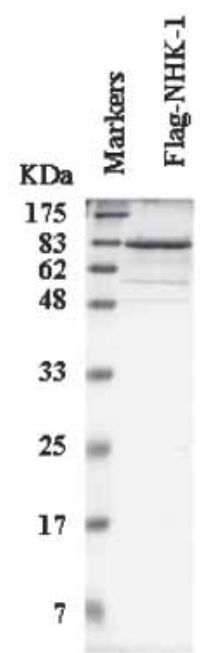

B

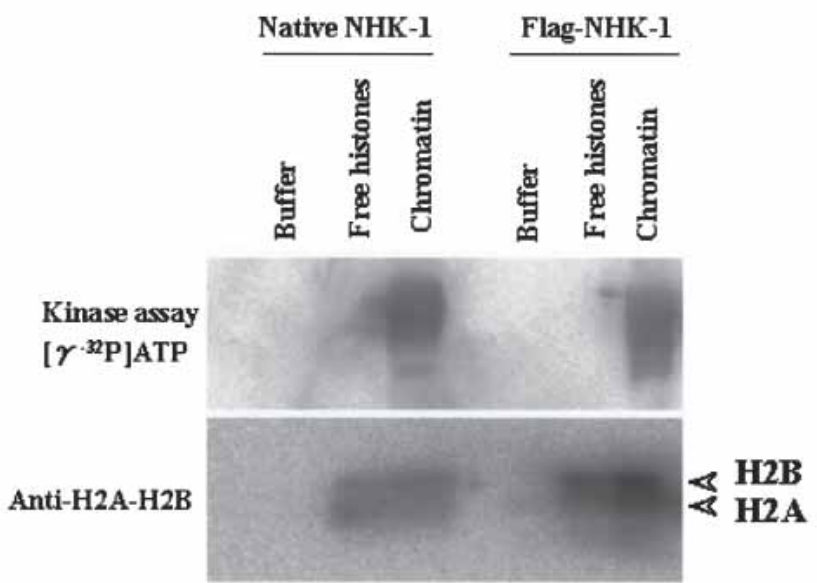

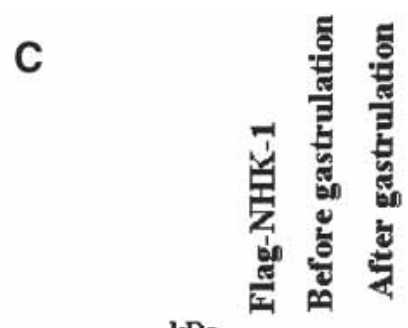

kDa

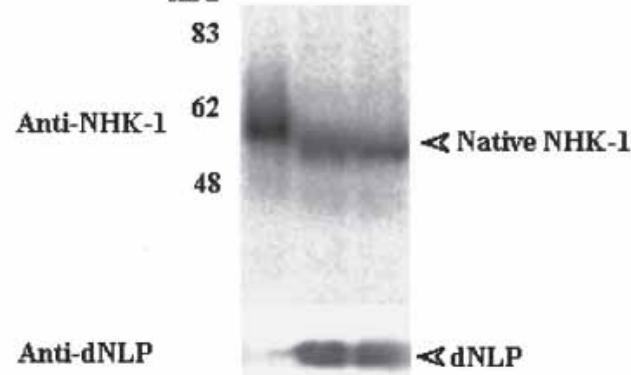

D

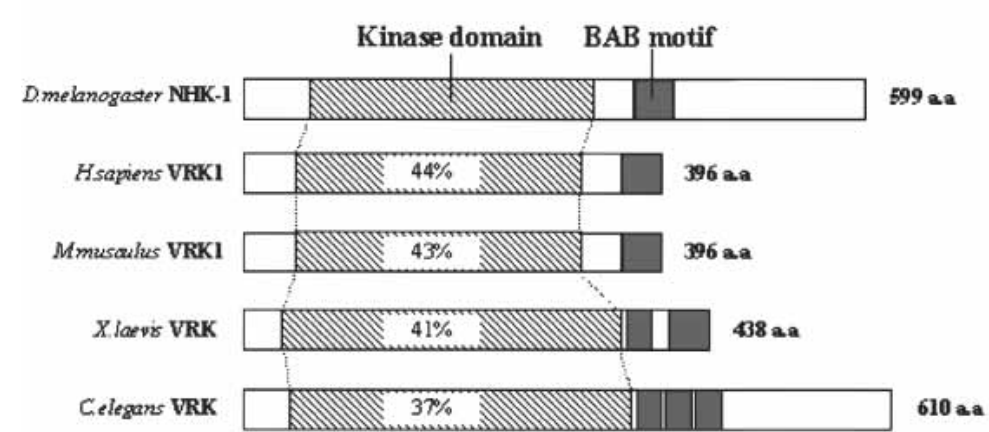

E

Figure 2. Purified recombinant NHK-1 phosphorylates nucleosomal H2A-H2B. $(A)$ Expression of recombinant Flag-tagged NHK-1 by E. coli. (B) Recombinant NHK-1 phosphorylates H2A-H2B in chromatin form. Kinase assay used native NHK-1 and Flag-tagged recombinant NHK-1 in the presence of free core histones or chromatin and $\left[\gamma_{-}{ }^{32} \mathrm{P}\right] \mathrm{ATP}$. For quantification of nucleosomes, anti-dH2A-H2B was used. (C) Equivalent amounts of the protein extracts $(20 \mu \mathrm{g})$ derived from Drosophila embryos before and after gastrulation were subjected to SDS-PAGE and Western blot analysis together with Flag-NHK-1. (Upper panel) The presence of NHK-1 detected with anti-NHK-1. (Lower panel) The presence of dNLP, which was proven to be expressed throughout development, to confirm the recovery of total protein (Ito et al. 1996a). (D) Schematic diagram of NHK-1 protein and its homologs; Homo sapiens VRK1 (Vaccinia related kinase 1), Mus musculus VRK1, Xenopus laevis VRK, and Caenorhabditis elegans VRK. The percent identity between each kinase and dNHK-1 over the kinase domain is shown in the boxes. (E) Alignment of basic-acidic-basic amino acid motif (BAB motif) in NHK-1 and counterparts of other homologs. Acidic and basic amino acids are shaded red or blue, respectively. 
A

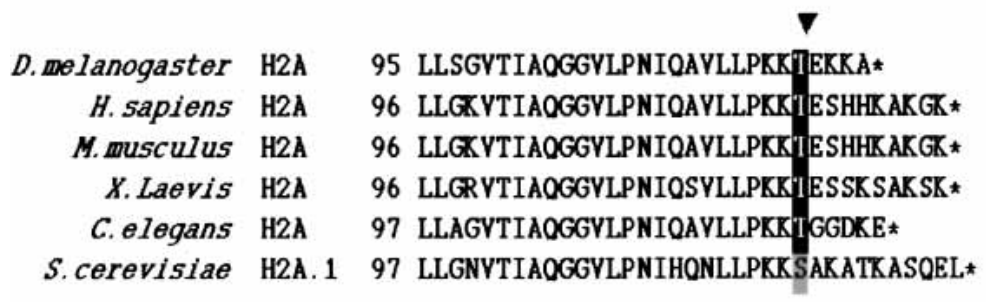

C

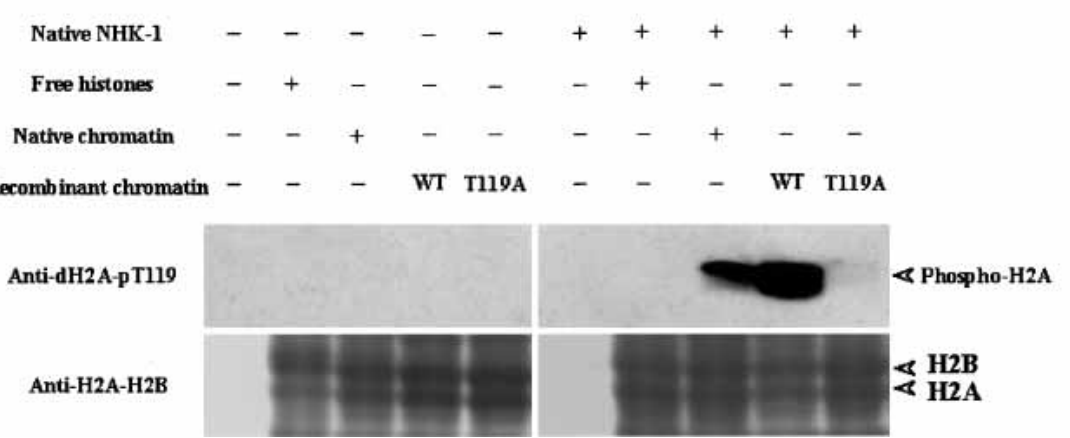

B

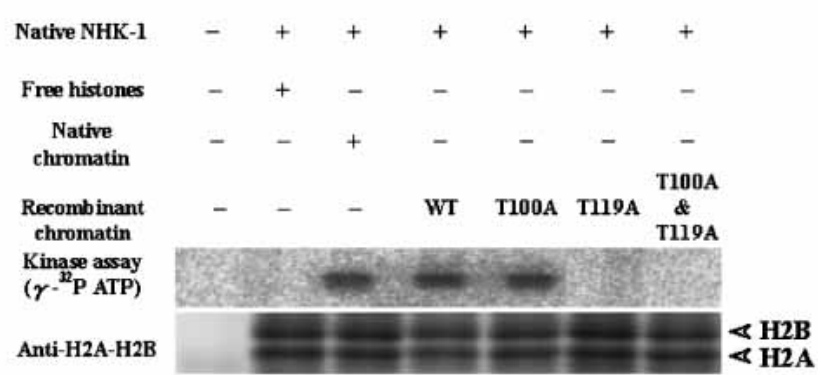

D

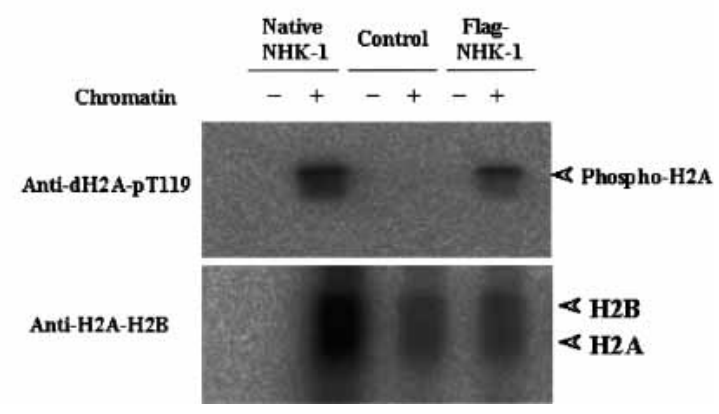

Figure 3. NHK-1 specifically phosphorylates Thr 119 of the histone H2A C terminus in vitro. (A) Sequence alignment of H2A C termini from Drosophila melanogaster, Homo sapiens, Mus musculus, Xenopus laevis, Caenorhabditis elegans, and Saccharomyces cerevisiae. The amino acid position in each protein is shown on the left. Stop codons are indicated by asterisks. The arrowhead indicates evolutionally conserved serine/threonine. (B) Drosophila native NHK-1 phosphorylates H2A Thr 119 specifically. A kinase assay was performed in the presence of $\left[\gamma^{-32} \mathrm{P}\right] \mathrm{ATP}$ using chromatin reconstituted with native core histones purified from Drosophila or recombinant core histones expressed by E. coli. (C) Antibodies raised against the phospho-Thr 119 H2A peptide specifically recognize phosphorylation of Drosophila Thr 119 of H2A. Kinase assay was done in the presence of core histones or equivalent amount of chromatin. $(D)$ Recombinant NHK-1 also phosphorylates Thr 119 of H2A. Chromatin was subjected to kinase assay using recombinant NHK-1 and native NHK-1. As control, partially purified nonspecific histone kinase, which phosphorylates only free core histones, was used. Phosphorylation was detected by Western blotting with anti-dH2A-pT119. T100A or T119A indicates that H2A Thr 100 or Thr 119 is substituted with alanine, respectively. (B,C) WT indicates chromatin reconstituted with wild-type H2A. $(B-D)$ Anti-dH2A-H2B was used for quantification of nucleosomes. 
gested with lysylendopeptidase followed by fractionation by HPLC. More than $90 \%$ of ${ }^{32} \mathrm{P}$ labeling is included in one fragment. Peptide sequencing analysis indicated that $\mathrm{H} 2 \mathrm{~A} \mathrm{C}$-terminal fragment residues were phosphorylated (LLSGVTIAQGGVLPNIQAVLLPKKTIEK in Fig. 3A). In addition, phosphopeptide mapping analysis revealed that threonine was phosphorylated (data not shown). These data suggest that Thr 100 and/or Thr 119 of the H2A C terminus is phosphorylated (Fig. 3A).

We then determined the specific phosphorylation site of NHK-1 using recombinant histone proteins expressed in E. coli. Chromatin containing Thr 100 substituted with alanine was phosphorylated by NHK-1 (Fig. 3B, T100A). However, chromatin with Ala 119 substituting for Thr 119 or alanines substituting for both Thr 100 and Thr 119 were not phosphorylated by NHK-1 (Fig. 3B, T119A, and T100A \& T119A). These experiments indicate that NHK-1 specifically phosphorylates threonine residue 119 of $\mathrm{H} 2 \mathrm{~A}$.

Because H2A and $\mathrm{H} 2 \mathrm{~B}$ make a dimer and run close together on SDS-PAGE, we usually refer to H2A-H2B as if they are a signal of the same size. In the earlier characterization, we referred to $\mathrm{H} 2 \mathrm{~A}-\mathrm{H} 2 \mathrm{~B}$ kinase because of the size of the signals and the band appearing to be double. However, from amino acid substitution experiments, we conclude that NHK-1 phosphorylates only Thr 119 in H2A specifically in vitro. The band appeared double partially because the high sensitivity of the assay might detect the small background.

This phosphorylation site and N-terminal proximal re- gions LLPKK(S/T) are highly conserved among eukaryotes from yeast to mammals (Fig. 3A). To examine this further, we raised rabbit polyclonal antibodies that recognize phospho-Thr 119 of Drosophila H2A /referred to as anti-dH2A-pT119). Phosphorylation of Thr 119 in H2A by NHK-1 was detected by immunoblotting analysis by using rabbit anti-dH2A-pT119 (Fig. 3C). It is noteworthy that Flag-tagged recombinant NHK-1 also phosphorylates Thr 119 in nucleosomal H2A (Fig. 3D).

Because chromatin, not free histones, is the substrate of NHK-1, NHK-1 might have a higher affinity to chromatin than to free histones. To examine this possibility, the interaction between recombinant Flag-tagged NHK-1 and DNA, free histones, or chromatin was analyzed by immunoprecipitation and subsequent elution with different salt concentrations. DNA and free histones did not bind to the NHK-1 affinity column (Fig. 4A,B), while chromatin remained bound to the NHK-1 column until it was eluted by $0.5 \mathrm{M} \mathrm{KCl}$ buffer (Fig. 4C). This result suggests that there is an interaction between NHK-1 and chromatin that does not occur between NHK-1 and free histones. There was no obvious difference of affinity between NHK-1 and wild-type recombinant chromatin or any mutant recombinant chromatin.

Previously, it was shown that $\mathrm{H} 2 \mathrm{~A}$ is phosphorylated in vivo (for review, see van Holde 1989). However, it is not known if Thr 119 in H2A is phosphorylated in vivo or what its physiological importance is. To examine these questions, we performed immunoblotting of Drosophila embryos using anti-dH2A-pT119. Drosophila
A Plasmid DNA

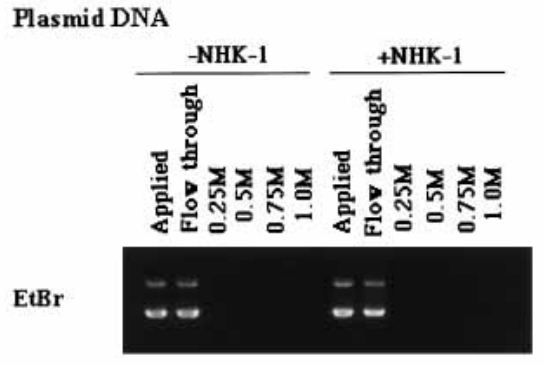

B Free core histone

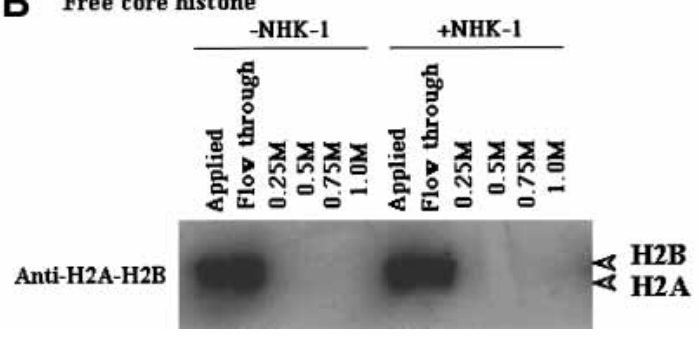

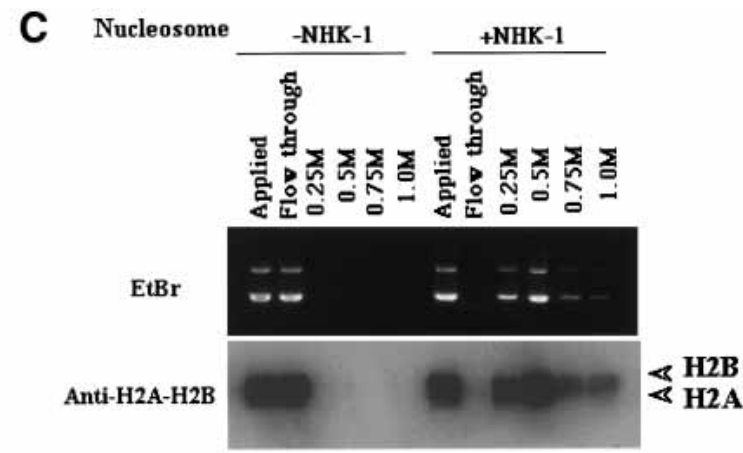

D

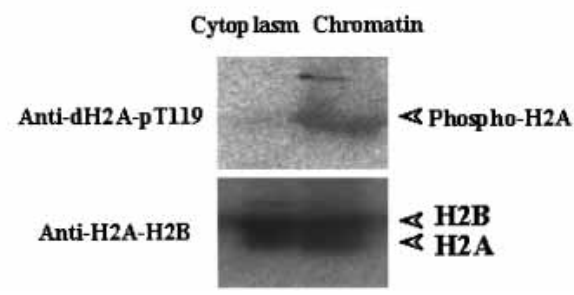

Figure 4. NHK-1 has higher affinity for chromatin than free histones. Recombinant NHK-1 was immobilized to resin and incubated with DNA $(A)$, free core histones $(B)$, or chromatin $(C)$. Affinity was examined by stepwise salt elution with $0.25,0.50,0.75$, and 1.0 $\mathrm{M} \mathrm{KCl}$. Each sample was analyzed by Western blotting with anti-H2A-H2B or agarose gel electrophoresis followed by EtBr staining. $(D)$ Thr 119 of histone H2A is phosphorylated in chromatin form but not in the free histone pool in vivo. The cytoplasmic fraction and chromatin fraction from 0-1-h Drosophila embryos were subjected to Western blotting with anti-dH2A-pT119 and anti-dH2A-H2B. 
embryos $(0-1 \mathrm{~h})$ were fractionated into cytoplasm and acid-soluble chromatin. Thr 119 of H2A from early-stage embryos was phosphorylated in the chromatin fraction but not in the cytoplasmic fraction (Fig. 4D). These results are consistent with the fact that NHK-1 phosphorylated Thr 119 of H2A only in chromatin form in vitro.

\section{NHK-1 activity is conserved from yeast to humans}

To examine conservation of NHK activity, we tested if Drosophila NHK-1 phosphorylates Thr 120 in human H2A (Fig. 5A). We performed a kinase assay using chromatin reconstituted with core histones derived from HeLa cells and recombinant dNHK-1. The reaction mixture was subjected to immunoblotting with anti-hH2ApT120 (Fig. 6A). The results show that dNHK-1 can phosphorylate Thr 120 of H2A in HeLa chromatin (corresponding to Thr 119 in Drosophila). These results suggest that the basic function of phosphorylation is conserved between mammals and Drosophila.

To confirm conservation among other species, we performed a kinase assay using an NHK activity that was partially purified from Saccharomyces cerevisiae extracts. A partially purified yeast NHK phosphorylated Thr 119 of H2A in Drosophila chromatin (Fig. 5B). Thus, in $S$. cerevisiae, there is a kinase that phosphorylates serine/threonine of the $\mathrm{H} 2 \mathrm{~A} \mathrm{C}$ terminus in chromatin form. These results indicate that NHK activity is conserved from yeast to humans, suggesting that this phosphorylation is important for basic cell functions.

Histone H2A is phosphorylated at Thr 119 during mitosis in Drosophila embryos

To characterize phosphorylation of H2A Thr 119 in vivo, we examined early-stage Drosophila embryos using antidH2A-pT119. After fertilization of the Drosophila embryo, the somatic nuclei in the syncytial egg undergo 13 consecutive and nearly synchronous divisions in the absence of G1 or G2 phase (Foe and Alberts 1983). For immunostaining, we used 0-1-h Drosophila embryos, which are at the stage prior to cellularization and gastrulation. Cycles 7-12 Drosophila embryos were ob- served, and typical immunostaining is shown in Figure 6. As shown in Figure 6A, there was no significant staining when phospho-peptide was used to block anti-dH2ApT119 as control. DNA staining with AcD is shown in Figure 6C, and merged features between Figure 6A and C are shown in Figure 6B. Using anti-dH2A-pT119, there is no significant staining in the early S-phase embryo, as shown in Figure 6D. However, chromatin begins to be stained with anti-dH2A-pT119 during cell cycle progression from $S$ phase to prophase, as shown in Figure 6G-L. Stained chromatin is more condensed compared with unstained chromatin, as shown in Figure $6 \mathrm{~K}$, which is an enlargement of the box in Figure $6 \mathrm{H}$. Chromatin is most strongly stained during metaphase, as shown in Figure 6M-O. Anaphase-to-telophase chromatin showed decreased immunostaining, suggesting that phosphorylated histone $\mathrm{H} 2 \mathrm{~A}$ at Thr 119 is dephosphorylated after mitosis (Fig. 6P-R). Because early-stage embryos have more mitotic nuclei compared with late-stage embryos, it is reasonable to observe that it was difficult to detect phosphorylation of Thr 119 of H2A with antibody in core histones of late-stage embryos or whole-stage embryos because of the decreased proportion of mitotic cells (data not shown). Thus, core histones purified from embryos up to $12 \mathrm{~h}$ old are relatively hypophosphorylated for Thr 119 of $\mathrm{H} 2 \mathrm{~A}$.

Immunostaining studies of Drosophila embryos clearly indicate that phosphorylation of Thr 119 is enhanced during mitosis and decreased during $S$ phase (Fig. 6 ), suggesting that this conserved phosphorylation site may play an important role in biological processes in the cell cycle, gene replication, or transcriptional regulation. This immunostaining pattern of phosphorylation of the conserved C-terminal residue, which is shown in Figure $3 \mathrm{~A}$, is detected in both early-stage embryos and cultured mammalian HeLa cells during mitosis $(\mathrm{H}$. Aihara and $\mathrm{T}$. Ito, unpubl.). It is suggested that this phosphorylation is a conserved phenomenon among species.

\section{Localization of NHK-1 in the Drosophila embryo}

To characterize NHK-1 in vivo, we examined the localization of NHK-1 in the early Drosophila embryo. For
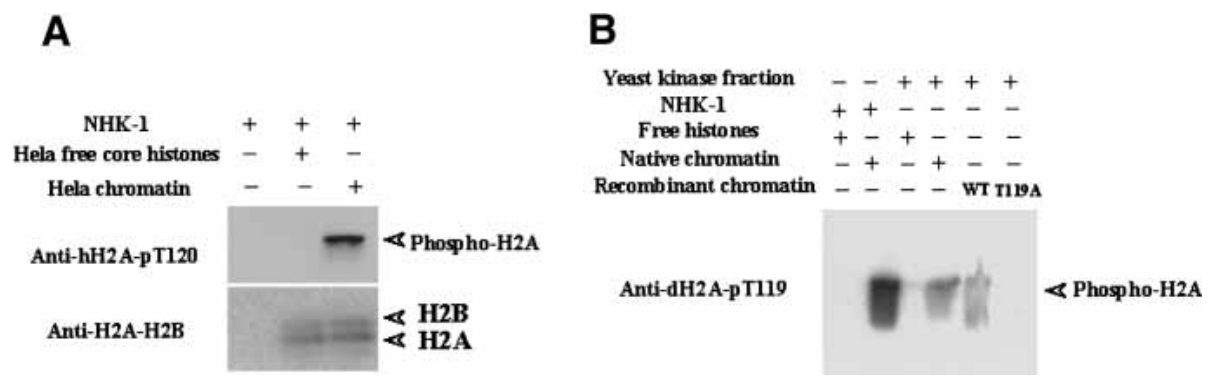

Figure 5. NHK-1 activity is conserved among species. (A) Recombinant dNHK-1 can phosphorylate human chromatin. The reaction used recombinant human NHK-1 and chromatin reconstituted with HeLa core histones or HeLa core histones only. The reaction mixture was subjected to Western blotting after incubation with cold ATP. (B) Yeast NHK phosphorylates Drosophila Thr 119 of H2A only in chromatin form. Partially purified yeast NHK with SP Sepharose chromatography and native dNHK-1 were subjected to kinase assay with anti-dH2A-pT119. 
Aihara et al.
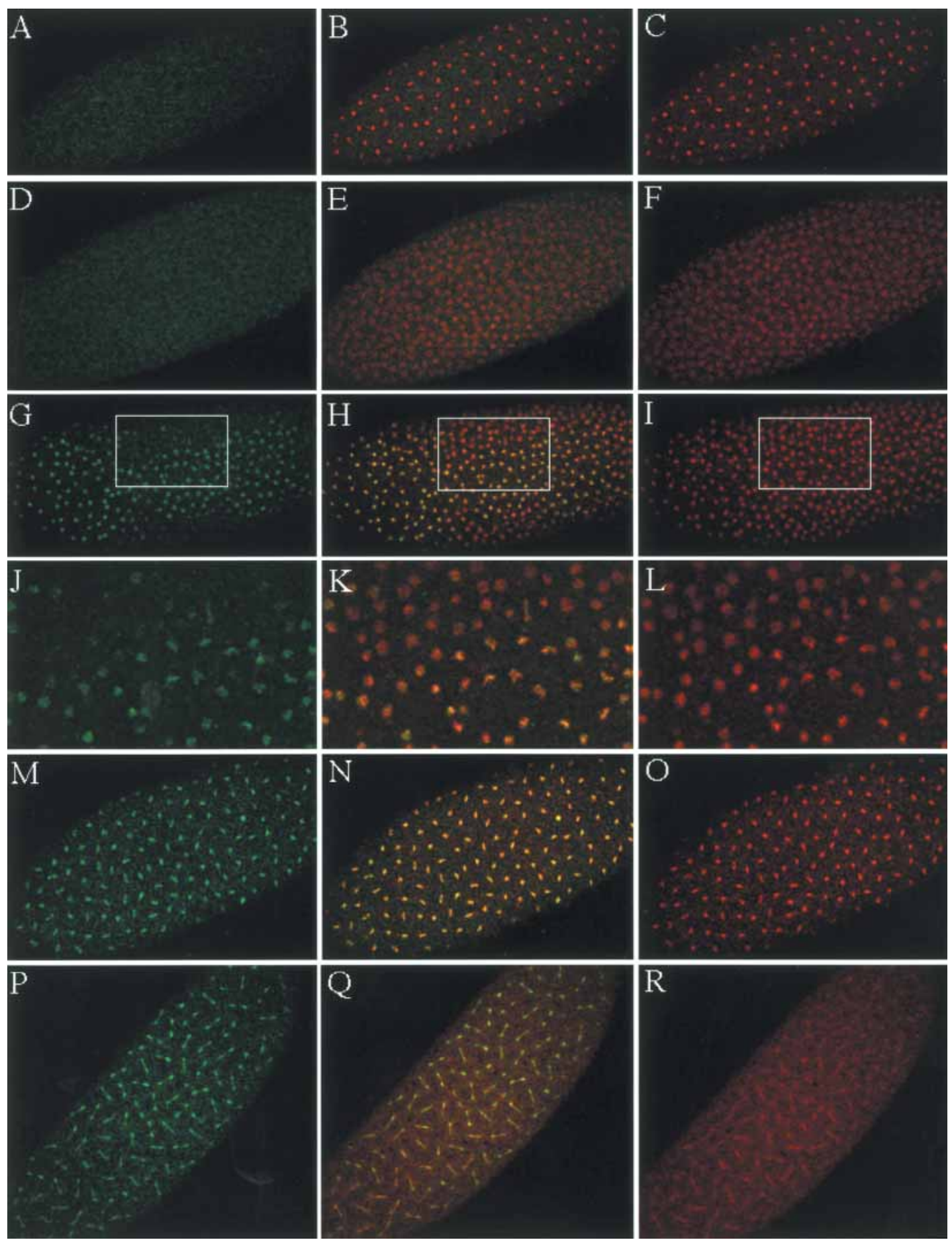

Figure 6. Histone H2A Thr 119 is phosphorylated during mitosis. $(A, D, G, J, M, P)$ For immunostaining, $0-1$-h Drosophila embryos were used. Drosophila embryos were immunostained with anti-dH2A-pT119. $(C, F, I, L, O, R)$ DNA was visualized with Actinomycin D. $(B, E, H, K, N, Q)$ Immunostaining with rabbit anti-dH2A-pT119 and DNA staining were merged, and overlapping is visualized by yellow. Immunostaining of $S$ phase $(D-F)$, prophase $(G-L)$, metaphase $(M-O)$, and anaphase and telophase $(P-R)$ are shown. $(A-C)$ Phosphopeptide was used to block anti-dH2A-pT119 as control. There was no significant nuclear staining when antibody is blocked with phosphopeptide as shown in $A$. Enlargements of the boxes in $G, H$, and $I$ are shown in $I, K$, and $L$, respectively.

immunostaining, we used 0-1-h Drosophila embryos, which are before cellularization and gastrulation. For immunostaining, we used rabbit anti-NHK-1, which is spe- cific for NHK-1, in immunoblotting analysis with the native crude extract as shown in Figure 2C. Cycle 7-12 Drosophila embryos were observed, and typical immu- 
nostaining is shown in Figure 7. In early S phase, NHK-1 mainly localized in the cytoplasm, as shown in Figure 7D. Localization of NHK-1 changes during cell cycle. The nuclei that are shown in Figure $7 \mathrm{I}$ are going into prophase. NHK-1 became localized to more condensed chromatin, as shown in yellow in the merged image (Fig. 7H) of NHK-1 staining (Fig. 7G) and DNA staining (Fig. 7I). During metaphase, NHK-1 stays in the nuclei, as shown in yellow by the merged image (Fig. $7 \mathrm{~K}, \mathrm{M}$ ) of NHK-1staining (Fig. 7J,M) and DNA staining (Fig. 7L,O). At the end of mitosis, NHK-1 is excluded from chromatin, as shown in red nuclei by the merged image (Fig. 7Q) of NHK-1 staining (Fig. 7P) and DNA staining (Fig. 7R). The fact that NHK-1 and DNA staining overlap at the peripheral region of the nuclei might reflect NHK-1 being pumped out at the nuclear membrane but is also
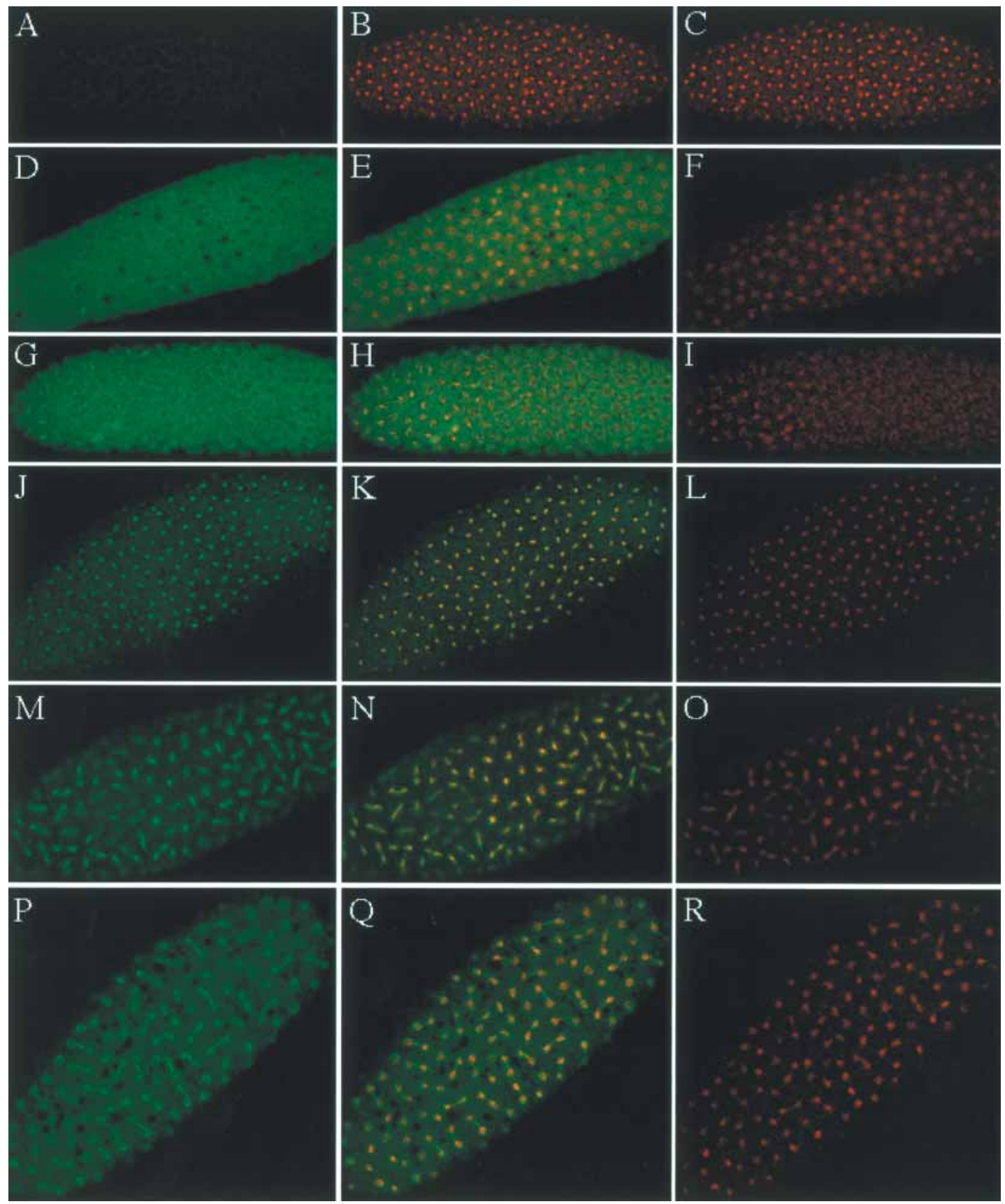

Figure 7. The localization of NHK-1 in the syncytial Drosophila embryo is changing depending on cell cycle. For immunostaining, 0-1-h Drosophila embryos were used. Drosophila embryos were immunostained with preimmune serum $(A)$ and rabbit anti-NHK-1 antibody $(D, G, J, M, P)$. $(C, F, I, L, O, R)$ DNA was visualized with Actinomycin D. $(B, E, H, K, N, I T L Q)$ Immunostaining with preimmune serum or anti-NHK-1 and DNA staining were merged, and overlapping is visualized by yellow. Immunostaining of $S$ phase $(D-F)$, prophase $(G-I)$, metaphase $(I-O)$, and anaphase and telophase $(P-R)$ is shown. 
partially because of the resolution of the microscope, given that the chromatin and cytoplasm are close, being separated by a thin nuclear membrane and matrix.

The cell cycle without G1 and G2 phase, which is shown in early Drosophila development, illustrates these changes of NHK-1 localization. They correlate well with the phosphorylation of H2A Thr 119 that also occurs during mitosis. These correlations between NHK-1 localization and the timing of phosphorylation, together with the strict substrate specificity of NHK-1 that only phosphorylates nucleosomal histone H2A in vitro, support the concept that H2A Thr 119 is the real substrate of NHK-1 in vivo. In addition, these immunostaining data suggest that NHK-1 plays an important role in cell cycle progression and other biological phenomena.

\section{Discussion}

Here we identify and characterize NHK-1, which phosphorylates a novel site, Thr 119, in the C terminus of H2A. We demonstrate that this site can be phosphorylated specifically in the nucleosome context. Furthermore, the physiological importance of this residue is suggested following study of immunostaining of early-stage embryos.

Recently, it was shown that the histone code is recognized by protein-protein interaction, for instance, HP-1 recognizes methylated Lys 9 of $\mathrm{H} 3$ (Bannister et al. 2001; Lachner et al. 2001). Thus, it is also possible that a unique protein recognizes the phosphorylated $\mathrm{C}$ terminus of $\mathrm{H} 2 \mathrm{~A}$ and is connected to the subsequent biological phenomena. As protein-protein interaction may link the histone code and phenotype (as HP-1 and H3 Lys 9 methylation), it is not merely enough to explain the phenotype of the yeast H2A C-terminal mutant completely by the electrical charge characteristic of the amino acid.

In addition, it has become clear that there is cross-talk between core histone modifications. For example, the choice of H3 Lys 9 methylation could be directed by H3 Ser 10 phosphorylation. In S. cerevisiae, Snf1 and GCN5 appear to work synergistically to mediate gene activation by phosphorylating $\mathrm{H} 3$ Ser 10 and acetylating H3 Lys 14, respectively (Lo et al. 2001). Another intriguing cross-talk linking ubiquitination of $\mathrm{H} 2 \mathrm{~B}$ to methylation of H3 was also shown in S. cerevisiae. Genetic experiments demonstrated a link between ubiquitination of H2B by Rad6/Ubc2 and the methylation of $\mathrm{H} 3$ on Lys 4 in budding yeast (Sun and Allis 2002).

Ubiquitination of H2A has also been known for a long time, but without understanding of its physiological role. H2A Lys 119, which is next to a unique phosphorylation site in mammals, is well conserved and known to be modified by mono ubiquitination (Olson et al. 1976; Goldknopf and Busch 1977). Busch and coworkers have shown that ubiquitinated H2A decreases markedly when rat liver nuclei were stimulated to synthesize rRNA by partial hepatectomy (Ballal et al. 1975). Although the physiological role of ubiquitination has remained unclear for a long time, understanding of H2A Thr 120 phosphorylation may uncover the role of ubiquitination of H2A Lys 119.

The shuttling of NHK-1 between cytoplasm and chromatin being dependent on cell cycle and the correlated phosphorylation of H2A Thr 119 in the chromatin suggests the physiological role of NHK-1 in cell cycle progression. Together with adjacent ubiquitination, which is known to change during hepatocyte proliferation, modification of the H2A C terminus might play a crucial role in cell proliferation. In addition, the transition between phosphorylation and dephosphorylation of this residue in $\mathrm{H} 2 \mathrm{~A}$ may change the structure of the nucleosome, the communication between histone code, or protein-protein interaction in the nucleus, thus leading to the regulation of transcription, DNA replication, and cell cycle progression.

\section{Materials and methods}

\section{Purification of NHK-1}

NHK-1 was purified from Drosophila embryos that were collected from 0 to $12 \mathrm{~h}$ after egg deposition, as shown in Figure 1B. A typical preparation of NHK-1 began with $2 \mathrm{~kg}$ of Drosophila embryos. NHK-1 was purified from nuclear extracts derived from embryos by ammonium sulfate precipitation and subsequent successive chromatographic steps on SP-Sepharose FF (Pharmacia), Q-Sepharose FF (Pharmacia), hydroxylapatite (BioRad), Blue Sepharose FF (Pharmacia), POROS Heparin (Boehringer Mannheim), Red Toyopearl (Tosoh), Sephacryl S-100 (Pharmacia), HiTrap Blue (Pharmacia), and Sephacryl S-200 (Pharmacia). The partial amino acid sequence of NHK-1 was determined principally as follows. The protein samples were subjected to electrophoresis on $10 \%$ SDS-PAGE and stained with Coomassie Brilliant Blue. The p70, p52, and p42 bands were excised and digested with lysylendopeptidase. The resulting peptides were purified by HPLC and sequenced with an automated protein sequencer (Applied Biosystems, Model cLc). Three independent runs of purification and subsequent peptide sequencing were done to ensure reproducibility of the data.

For the detailed methodology of NHK-1 purification, see Supplemental Material.

\section{Purification of recombinant NHK-1}

Tandemly connected Flag-tagged NHK-1 (Flag-NHK-1) was produced in E. coli strain Rosseta (DE3) pLysS (Invitrogen). A lysate of $E$. coli in which Flag-tagged NHK-1 was expressed was immunopurified with Flag M2 agarose (Sigma). After extensive washing of the agarose, Flag-tagged NHK-1 was eluted with Flag peptides (Sigma) and dialyzed against $50 \mathrm{mM} \mathrm{KCl}$.

\section{Reconstitution of nucleosomes}

Chromatin was reconstituted with plasmid DNA and purified Drosophila core histones by using salt dialysis techniques as described previously (Ito et al. 1997). The most highly reconstituted chromatin was then purified by $15 \%-40 \%$ glycerol gradient sedimentation $160,000 \mathrm{rpm}$ for $2.5 \mathrm{~h}$ at $4^{\circ} \mathrm{C}$; Beckman SW60 rotor) Recombinant histone proteins were expressed and purified as described previously (Levenstein and Kadonaga 2002).

\section{In vitro phosphorylation}

For the kinase assay, typically $1 \mathrm{fM}$ native NHK-1 or $100 \mathrm{fM}$ recombinant NHK-1 was incubated with $20 \mathrm{ng}$ of DNA, $10 \mathrm{ng}$ of 
H2A-H2B dimer, 20 ng of all four core histones, 30 ng of H2A$\mathrm{H} 2 \mathrm{~B} / \mathrm{DNA}$, or $40 \mathrm{ng}$ of chromatin as substrates in the presence of $19 \mathrm{kBq}$ of $\left[\gamma_{-}{ }^{32} \mathrm{P}\right] \mathrm{ATP}, 10 \mathrm{mM}$ HEPES $(\mathrm{pH} 7.6), 50 \mathrm{mM} \mathrm{KCl}$, and $5 \mathrm{mM} \mathrm{MgCl}$ for $2 \mathrm{~h}$ at $30^{\circ} \mathrm{C}$. Reaction mixtures were resolved by $15 \%$ SDS-PAGE and subjected to autoradiography. For immunoblotting with anti-dH2A-pT119 or anti-hH2ApT120, $1 \mathrm{nM}$ cold ATP was used instead of $\left[\gamma^{-32} \mathrm{P}\right] \mathrm{ATP}$ in the $50-\mu \mathrm{L}$ reaction mixture.

\section{In vitro interaction assay}

The NHK-1 affinity column was prepared by binding Flagtagged NHK-1 to Flag M2 agarose gel. DNA, free core histones, or chromatin was incubated with the affinity column under 50 $\mathrm{mM} \mathrm{KCl}, 5 \mathrm{mM} \mathrm{MgCl}_{2}$ conditions followed by stepwise elution with $0.25,0.50,0.75$, and $1.0 \mathrm{M} \mathrm{KCl}$. Eluates were precipitated with trichloroacetic acid (TCA) and subjected to $15 \%$ SDSPAGE and subsequent immunoblotting.

\section{Immunostaining of Drosophila embryos}

Drosophila embryos (collected from 0 to $1 \mathrm{~h}$ after egg deposition) were dechorionized and suspended in buffer containing 10 $\mathrm{mM}$ potassium phosphate (pH 6.8), $15 \mathrm{mM} \mathrm{NaCl}, 45 \mathrm{mM} \mathrm{KCl}$, and $2 \mathrm{mM} \mathrm{MgCl}_{2}$. The embryos were then permeabilized principally as described before (Ito et al. 1996b). After blocking with $10 \%$ BSA, the embryos were incubated with the primary antibody (anti-dH2A-pT119 or anti-NHK-1) overnight at $4^{\circ} \mathrm{C}$. The embryos were washed, and the secondary antibody (anti-rabit IgG conjugated with Alexa), along with $4 \mu \mathrm{g} / \mathrm{mL}$ Actinomycin D (for DNA staining), was incubated with the embryos overnight at $4^{\circ} \mathrm{C}$. The embryos were washed and then analyzed by confocal microscopy. Confocal images were collected by using a Carl Zeiss LSM 510 META confocal scanning laser head attached to a Carl Zeiss Axiovert $200 \mathrm{M}$ microscope. The data shown were representative of the consistently and reproducibly observed characteristics of many embryos.

\section{Acknowledgments}

We thank Jim Kadonaga and Toshifumi Matsuyama for helpful discussions and comments. We are grateful to Fred Winston for the yeast strain FY406 and the pJH55 plasmid and Mark Levenstein for the core histone plasmid. We also thank Tsuyoshi Ikehara and Shingo Yasuda for helpful discussions and technical support.

The publication costs of this article were defrayed in part by payment of page charges. This article must therefore be hereby marked "advertisement" in accordance with 18 USC section 1734 solely to indicate this fact.

\section{References}

Anest, V., Hanson, J.L., Cogswell, P.C., Steinbrecher, K.A., Strahl, B.D., and Baldwin, A.S. 2003. A nucleosomal function for ІкB kinase- $\alpha$ in NF-кB-dependent gene expression. Nature 423: 659-663.

Ballal, N.R., Kang, Y.J., Olson, M.O., and Busch, H. 1975. Changes in nucleolar proteins and their phosphorylation patterns during liver regeneration. J. Biol. Chem. 250: 59215925.

Bannister, A.J., Zegerman, P., Partridge, J.F., Miska, E.A., Thomas, J.O., Allshire, R.C., and Kouzarides, T. 2001. Selective recognition of methylated lysine 9 on histone H3 by the HP1 chromo domain. Nature 410: 120-124.
Carrozza, M.J., Utley, R.T., Workman, J.L., and Cote, J. 2003. The diverse functions of histone acetyltransferase complexes. Trends Genet. 19: 321-329.

Cheung, P., Tanner, K.G., Cheung, W.L., Sassone-Corsi, P., Denu, J.M., and Allis, C.D. 2000. Synergistic coupling of histone $\mathrm{H} 3$ phosphorylation and acetylation in response to epidermal growth factor stimulation. Mol. Cell 5: 905-915.

Cheung, W.L., Ajiro, K., Samejima, K., Kloc, M., Cheung, P., Mizzen, C.A., Beeser, A., Etkin, L.D., Chernoff, J., Earnshaw, W.C., et al. 2003. Apoptotic phosphorylation of histone H2B is mediated by mammalian sterile twenty kinase. Cell 113: $507-517$.

Downs, J.A., Lowndes, N.F., and Jackson, S.P. 2000. A role for Saccharomyces cerevisiae histone $\mathrm{H} 2 \mathrm{~A}$ in DNA repair. $\mathrm{Na}$ ture 408: 1001-1004.

Fischle, W., Wang, Y., and Allis, C.D. 2003. Histone and chromatin cross-talk. Curr. Opin. Cell Biol. 15: 172-183.

Foe, V.E. and Alberts, B.M. 1983. Studies of nuclear and cytoplasmic behaviour during the five mitotic cycles that precede gastrulation in Drosophila embryogenesis. J. Cell Sci. 61: $31-70$.

Goldknopf, I.L. and Busch, H. 1977. Isopeptide linkage between nonhistone and histone $2 \mathrm{~A}$ polypeptides of chromosomal conjugate-protein A24. Proc. Natl. Acad. Sci. 74: 864-868.

Goll, M.G. and Bestor, T.H. 2002. Histone modification and replacement in chromatin activation. Genes \& Dev. 16: $1739-1742$.

Hsu, J.Y., Sun, Z.W., Li, X., Reuben, M., Tatchell, K., Bishop, D.K., Grushcow, J.M., Brame, C.J., Caldwell, J.A., Hunt, D.F., et al. 2000. Mitotic phosphorylation of histone H3 is governed by Ipl1/aurora kinase and Glc7/PP1 phosphatase in budding yeast and nematodes. Cell 102: 279-291.

Ito, T. 2003. Nucleosome assembly and remodeling. Curr. Top. Microbiol. Immunol. 274: 1-22.

Ito, T., Tyler, J.K., Bulger, M., Kobayashi, R., and Kadonaga, J.T. 1996a. ATP-facilitated chromatin assembly with a nucleoplasmin-like protein from Drosophila melanogaster. I. Biol. Chem. 271: 25041-25048.

Ito, T., Bulger, M., Kobayashi, R., and Kadonaga, J.T. $1996 \mathrm{~b}$. Drosophila NAP-1 is a core histone chaperone that functions in ATP-facilitated assembly of regularly spaced nucleosomal arrays. Mol. Cell. Biol. 16: 3112-3124.

Ito, T., Bulger, M., Pazin, M.J., Kobayashi, R., and Kadonaga, J.T. 1997. ACF, an ISWI-containing and ATP-utilizing chromatin assembly and remodeling factor. Cell 90: 145-155.

Kamath, R.S., Fraser, A.G., Dong, Y., Poulin, G., Durbin, R., Gotta, M., Kanapin, A., Le Bot, N., Moreno, S., Sohrmann, M., et al. 2003. Systematic functional analysis of the Caenorhabditis elegans genome using RNAi. Nature 421: 231237.

Kornberg, R.D. and Lorch, Y. 1999. Twenty-five years of the nucleosome, fundamental particle of the eukaryote chromosome. Cell 98: 285-294.

Kurdistani, S.K. and Grunstein, M. 2003. Histone acetylation and deacetylation in yeast. Nat. Rev. Mol. Cell Biol. 4: 276284.

Lachner, M., O'Carroll, D., Rea, S., Mechtler, K., and Jenuwein, T. 2001. Methylation of histone H3 lysine 9 creates a binding site for HP1 proteins. Nature 410: 116-120.

Levenstein, M.E. and Kadonaga, J.T. 2002. Biochemical analysis of chromatin containing recombinant Drosophila core histones. J. Biol. Chem. 277: 8749-8754.

Lo, W.S., Trievel, R.C., Rojas, J.R., Duggan, L., Hsu, J.Y., Allis, C.D., Marmorstein, R., and Berger, S.L. 2000. Phosphorylation of serine 10 in histone $\mathrm{H} 3$ is functionally linked in vitro and in vivo to Gcn5-mediated acetylation at lysine 14. Mol. 
Aihara et al.

Cell 5: 917-926.

Lo, W.S., Duggan, L., Emre, N.C., Belotserkovskya, R., Lane, W.S., Shiekhattar, R., and Berger, S.L. 2001. Snf1-A histone kinase that works in concert with the histone acetyltransferase Gcn5 to regulate transcription. Science 293: 1142-1146.

Lopez-Borges, S. and Lazo, P.A. 2000. The human Vaccinia-related kinase 1 (VRK1) phosphorylates threonine-18 within the $\mathrm{mdm}-2$ binding site of the p53 tumour suppressor protein. Oncogene 19: 3656-3664.

Martens, J.A. and Winston, F. 2003. Recent advances in understanding chromatin remodeling by Swi/Snf complexes. Curr. Opin. Genet. Dev. 13: 136-142.

Narlikar, G.J., Fan, H.Y., and Kingston, R.E. 2002. Cooperation between complexes that regulate chromatin structure and transcription. Cell 108: 475-487.

Nezu, J., Oku, A., Jones, M.H., and Shimane, M. 1997. Identification of two novel human putative serine/threonine kinases, VRK1 and VRK2, with structural similarity to Vaccinia virus B1R kinase. Genomics 45: 327-331.

Olson, M.O., Goldknopf, I.L., Guetzow, K.A., James, G.T., Hawkins, T.C., Mays-Rothberg, C.J., and Busch, H. 1976. The $\mathrm{NH}_{2}$ - and $\mathrm{COOH}$-terminal amino acid sequence of nuclear protein A24. J. Biol. Chem. 251: 5901-5903.

Orphanides, G. and Reinberg, D. 2002. A unified theory of gene expression. Cell 108: 439-451.

Piano, F., Schetter, A.J., Morton, D.G., Gunsalus, K.C., Reinke, V., Kim, S.K., and Kemphues, K.J. 2002. Gene clustering based on RNAi phenotypes of ovary-enriched genes in $C$. elegans. Curr. Biol. 12: 1959-1964.

Rogakou, E.P., Nieves-Neira, W., Boon, C., Pommier, Y., and Bonner, W.M. 2000. Initiation of DNA fragmentation during apoptosis induces phosphorylation of H2AX histone at serine 139. J. Biol. Chem. 275: 9390-9395.

Sassone-Corsi, P., Mizzen, C.A., Cheung, P., Crosio, C., Monaco, L., Jacquot, S., Hanauer, A., and Allis, C.D. 1999. Requirement of Rsk-2 for epidermal growth factor-activated phosphorylation of histone H3. Science 285: 886-891.

Strahl, B.D. and Allis, C.D. 2000. The language of covalent histone modifications. Nature 403: 41-45.

Sun, Z.W. and Allis, C.D. 2002. Ubiquitination of histone H2B regulates $\mathrm{H} 3$ methylation and gene silencing in yeast. $\mathrm{Na}$ ture 418: 104-108.

van Holde, K.E. 1989. Chromatin. Springer-Verlag, New York.

Vignali, M., Hassan, A.H., Neely, K.E., and Workman, J.L. 2000. ATP-dependent chromatin-remodeling complexes. Mol. Cell. Biol. 20: 1899-1910.

Ward, I.M. and Chen, J. 2001. Histone H2AX is phosphorylated in an ATR-dependent manner in response to replicational stress. J. Biol. Chem. 276: 47759-47762.

Yamamoto, Y., Verma, U.N., Prajapati, S., Kwak, Y.T., and Gaynor, R.B. 2003. Histone H3 phosphorylation by IKK- $\alpha$ is critical for cytokine-induced gene expression. Nature 423: 655-659.

Zhang, Y. and Reinberg, D. 2001. Transcription regulation by histone methylation: Interplay between different covalent modifications of the core histone tails. Genes \& Dev. 15: 2343-2360. 


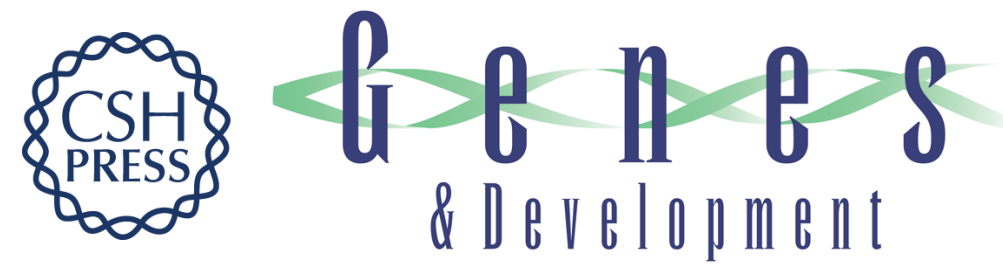

\section{Nucleosomal histone kinase-1 phosphorylates H2A Thr 119 during mitosis in the early Drosophila embryo}

Hitoshi Aihara, Takeya Nakagawa, Kiyoshi Yasui, et al.

Genes Dev. 2004, 18:

Access the most recent version at doi:10.1101/gad.1184604

Supplemental http://genesdev.cshlp.org/content/suppl/2004/04/12/1184604.DC1
Material

References This article cites 38 articles, 14 of which can be accessed free at:

http://genesdev.cshlp.org/content/18/8/877.full.html\#ref-list-1

License

Email Alerting Receive free email alerts when new articles cite this article - sign up in the box at the top

Service right corner of the article or click here.

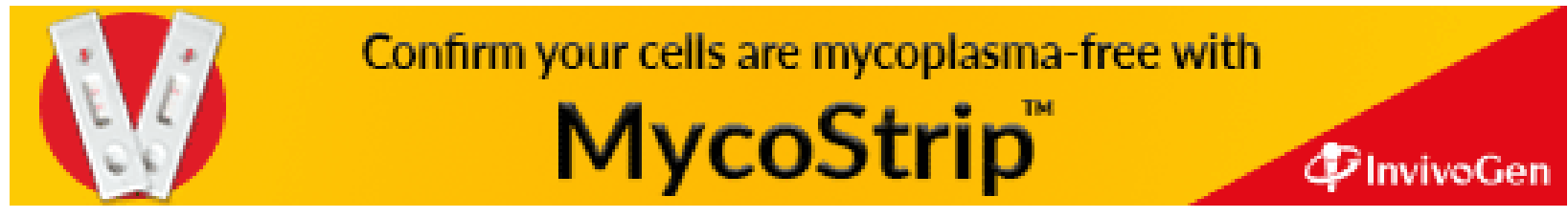

